\title{
Generalized Srivastava's triple hypergeometric functions and their associated properties
}

\author{
Junesang Choij ${ }^{a, *}$, Rakesh Kumar Parmar ${ }^{b}$ \\ ${ }^{a}$ Department of Mathematics, Dongguk University, Gyeongju 38066, Republic of Korea. \\ ${ }^{b}$ Department of Mathematics, Government College of Engineering and Technology, Bikaner 334004, Rajasthan, India.
}

Communicated by Y. J. Cho

\begin{abstract}
The main object of this paper is to introduce generalized Srivastava's triple hypergeometric functions by using the generalized Pochhammer symbol and investigate certain properties, for example, their various integral representations, derivative formulas and recurrence relations. Various (known or new) special cases and consequences of the results presented here are also considered. (C)2017 All rights reserved.
\end{abstract}

Keywords: Gamma function, beta function, generalized Pochhammer symbol, generalized hypergeometric function, extended Appell functions, generalized Srivastava's triple hypergeometric functions, Whittaker function, Bessel and modified Bessel functions.

2010 MSC: 33B15, 33B20, 33C05, 33C15, 33C20, 33B99, 33C99, $60 \mathrm{~B} 99$.

\section{Introduction, definitions and preliminaries}

In recent years, various extensions of the well-known special functions have been presented and investigated (see, e.g., [3-5, 11, 12, 28]). In particular, Chaudhry and Zubair [1] (see also [2]) introduced an interesting generalization of the familiar gamma function $\Gamma(z)$ (see, e.g., [25, Section 1.1]) by inserting the regularization factor $\exp \left(-\frac{p}{t}\right)$ as follows:

$$
\Gamma_{p}(z):= \begin{cases}\int_{0}^{\infty} t^{z-1} \exp \left(-t-\frac{p}{t}\right) d t, & (\mathfrak{R}(p)>0, \quad z \in \mathbb{C}), \\ \Gamma(z), & (p=0, \mathfrak{R}(z)>0) .\end{cases}
$$

Here and in the following, let $\mathbb{N}, \mathbb{Z}^{-}$and $\mathbb{C}$ be the sets of positive integers, negative integers and complex numbers, respectively and let $\mathbb{N}_{0}:=\mathbb{N} \cup\{0\}$ and $\mathbb{Z}_{0}^{-}:=\mathbb{Z}^{-} \cup\{0\}$.

Recently, Srivastava et al. [23, p. 487, Eq. (15)] have introduced and studied in a rather systematic manner the following generalized hypergeometric function:

$$
{ }_{r} F_{s}\left[\begin{array}{r}
\left(\alpha_{1}, p\right), \alpha_{2}, \cdots, \alpha_{r} ; z \\
\beta_{1}, \cdots, \beta_{s} ;
\end{array}\right]=\sum_{n=0}^{\infty} \frac{\left(\alpha_{1} ; p\right)_{n}\left(\alpha_{2}\right)_{n} \cdots\left(\alpha_{r}\right)_{n}}{\left(\beta_{1}\right)_{n} \cdots\left(\beta_{s}\right)_{n}} \frac{z^{n}}{n !},
$$

\footnotetext{
*Corresponding author

Email addresses: junesang@mail.dongguk.ac.kr (Junesang Choi), rakeshparmar27@gmail.com (Rakesh Kumar Parmar)
} doi:10.22436/jnsa.010.02.41 
where $(\lambda ; p)_{v}$ is the generalized Pochhammer symbol defined by (see [23, p. 485, Eq. (8)]):

$$
(\lambda ; p)_{v}:= \begin{cases}\frac{\Gamma_{p}(\lambda+v)}{\Gamma(\lambda)}, & (\mathfrak{R}(p)>0, \quad \lambda, v \in \mathbb{C}), \\ (\lambda)_{v}, & (p=0, \lambda, v \in \mathbb{C})\end{cases}
$$

and $(\lambda)_{v}(\lambda, v \in \mathbb{C})$ is the familiar Pochhammer symbol (or the shifted factorial) defined (in general) by

$$
(\lambda)_{v}:=\frac{\Gamma(\lambda+v)}{\Gamma(\lambda)}= \begin{cases}1, & (v=0, \quad \lambda \in \mathbb{C} \backslash\{0\}), \\ \lambda(\lambda+1) \cdots(\lambda+n-1), & (v=n \in \mathbb{N}, \quad \lambda \in \mathbb{C})\end{cases}
$$

it being understood conventionally that $(0)_{0}:=1$ and assumed tacitly that the $\Gamma$-quotient exists (see, for details, [26, p. 21 et seq.]). The integral representation of $(\lambda ; p)_{v}$ is given as follows (see [23, p. 485, Eq. (9)]):

$$
\begin{aligned}
& (\lambda ; p)_{v}=\frac{1}{\Gamma(\lambda)} \int_{0}^{\infty} t^{\lambda+v-1} \exp \left(-t-\frac{p}{t}\right) d t \\
& (\mathfrak{R}(p)>0, \quad \mathfrak{R}(\lambda+v)>0, \quad \text { when } \quad p=0) .
\end{aligned}
$$

Clearly, when $p=0,(1.2)$ reduces to the (1.3) and (1.1) reduces to the familiar generalized hypergeometric function ${ }_{r} F_{s}$ (see, e.g., $\left.[13,15]\right)$.

Very recently, Srivastava et al. [29] have introduced and studied three extended Lauricella functions $F_{A}^{(n)}, F_{C}^{(n)}$ and $F_{D}^{(n)}$ of $n$ variables together with those in their obvious special cases when $n=2$ as for the corresponding Appell functions $F_{2}, F_{4}$ and $F_{3}$, respectively. For our present investigation, we recall the extended second Appell function $F_{2}$ (see [29, p. 153, Eq. (2.2)]):

$$
\begin{gathered}
F_{2}\left[(\alpha, p), \beta_{1}, \beta_{2} ; \gamma_{1}, \gamma_{2} ; x_{1}, x_{2}\right]=\sum_{m_{1}, m_{2}=0}^{\infty} \frac{(\alpha ; p)_{m_{1}+m_{2}}\left(\beta_{1}\right)_{m_{1}}\left(\beta_{2}\right)_{m_{2}}}{\left(\gamma_{1}\right)_{m_{1}}\left(\gamma_{2}\right)_{m_{2}}} \frac{x_{1}^{m_{1}}}{m_{1} !} \frac{x_{2}^{m_{2}}}{m_{2} !}, \\
\left(\Re(p) \geqq 0, \quad\left|x_{1}\right|+\left|x_{2}\right|<1, \quad \text { when } p=0\right),
\end{gathered}
$$

and two of the confluent forms of Appell series in two variables defined by

$$
\Psi_{2}\left[a ; c_{1}, c_{2} ; x_{1}, x_{2}\right]=\sum_{m, n=0}^{\infty} \frac{(a)_{m+n}}{\left(c_{1}\right)_{m}\left(c_{2}\right)_{n}} \frac{x_{1}^{m}}{m !} \frac{x_{2}^{n}}{n !}, \quad\left(\left|x_{1}\right|<\infty,\left|x_{2}\right|<\infty\right),
$$

(see, e.g., [26, p.26, Eq. (22)]) and

$$
\Phi_{3}\left[a ; c ; x_{1}, x_{2}\right]=\sum_{m, n=0}^{\infty} \frac{(a)_{m}}{(c)_{m+n}} \frac{x_{1}{ }^{m}}{m !} \frac{x_{2}^{n}}{n !}, \quad\left(\left|x_{1}\right|<\infty,\left|x_{2}\right|<\infty\right),
$$

(see, e.g., [26, p.26, Eq. (20)]).

Motivated mainly by some of these aforementioned investigations of the extended special functions, here, we aim to introduce the generalizations of the Srivastava's triple hypergeometric functions $H_{A}, H_{B}$ and $\mathrm{H}_{\mathrm{C}}$ (see, e.g., [26, p.43, Eq. (12)]; see also [27, p.68, Eq. (37)]) and systematically investigate various properties of the generalized Srivastava's triple hypergeometric functions $H_{A}, H_{B}$ and $H_{C}$, for example, integral representations, some of which contains Laguerre polynomial, Whittaker function, Bessel and modified Bessel functions in the kernel, derivative formulas and recurrence relations.

For various other investigations involving generalizations of the hypergeometric function ${ }_{r} F_{s}$ of $r$ numerator and $s$ denominator parameters, which were motivated essentially by the work of Srivastava et al. [23], the interested reader may be referred to several recent papers on the subject (see, e.g., [10, 21-24] and the references cited therein). 


\section{Generalizations of the Srivastava's triple hypergeometric functions}

In terms of the generalized Pochhammer symbol in (1.2), we generalize the Srivastava's triple hypergeometric functions $\mathrm{H}_{\mathrm{A}}, \mathrm{H}_{B}$ and $\mathrm{H}_{C}$ as follows:

$$
\begin{gathered}
\mathrm{H}_{\mathrm{A}}\left[(\alpha, p), \beta_{1}, \beta_{2} ; \gamma_{1}, \gamma_{2} ; x_{1}, x_{2}, x_{3}\right]=\sum_{l, m, n}^{\infty} \frac{(\alpha ; p)_{l+n}\left(\beta_{1}\right)_{l+m}\left(\beta_{2}\right)_{m+n}}{\left(\gamma_{1}\right)_{l}\left(\gamma_{2}\right)_{m+n}} \frac{x_{1}^{l}}{l !} \frac{x_{2}^{m}}{m !} \frac{x_{3}^{n}}{n !}, \\
\left(p \geqq 0, \quad\left|x_{1}\right|<r, \quad\left|x_{2}\right|<s, \quad\left|x_{3}\right|<t, \quad r+s+t=1+s t \text { when } p=0\right), \\
H_{B}\left[(\alpha, p), \beta_{1}, \beta_{2} ; \gamma_{1}, \gamma_{2}, \gamma_{3} ; x_{1}, x_{2}, x_{3}\right]=\sum_{l, m, n}^{\infty} \frac{(\alpha ; p)_{l+n}\left(\beta_{1}\right)_{l+m}\left(\beta_{2}\right)_{m+n}}{\left(\gamma_{1}\right)_{l}\left(\gamma_{2}\right)_{m}\left(\gamma_{3}\right)_{n}} \frac{x_{1}^{l}}{l !} \frac{x_{2}^{m}}{m !} \frac{x_{3}^{n}}{n !}, \\
\left(p \geqq 0, \quad\left|x_{1}\right|<r, \quad\left|x_{2}\right|<s, \quad\left|x_{3}\right|<t, \quad r+s+t+2 \sqrt{r s t}=1 \text { when } p=0\right),
\end{gathered}
$$

and

$$
\begin{gathered}
H_{C}\left[(\alpha, p), \beta_{1}, \beta_{2} ; \gamma ; x_{1}, x_{2}, x_{3}\right]=\sum_{l, m, n=0}^{\infty} \frac{(\alpha ; p)_{l+n}\left(\beta_{1}\right)_{l+m}\left(\beta_{2}\right)_{m+n}}{(\gamma)_{l+m+n}} \frac{x_{1}^{l}}{l !} \frac{x_{2}^{m}}{m !} \frac{x_{3}^{n}}{n !}, \\
\left(p \geqq 0, \quad\left|x_{1}\right|<r, \quad\left|x_{2}\right|<s, \quad\left|x_{3}\right|<t, \quad r+s+t-2 \sqrt{(1-r)(1-s)(1-t)}<2 \text { when } p=0\right),
\end{gathered}
$$

where $\alpha, \beta_{1}, \beta_{2} \in \mathbb{C}$ and $\gamma, \gamma_{1}, \gamma_{2}, \gamma_{3} \in \mathbb{C} \backslash \mathbb{Z}_{0}^{-}$.

Remark 2.1. Clearly, when $p=0,(2.1),(2.2)$ and (2.3) reduce to the Srivastava's triple hypergeometric functions (see, e.g., [26, p.43, Eq. (12)] and [27, p.68, Eq. (37)], see also [16-20]). The special cases of (2.1) and (2.2) when $x_{2}=0$ reduce to the extended second Appell's hypergeometric functions $F_{2}$ in (1.5). When $x_{2}=0,(2.3)$ gives the extended first Appell's hypergeometric function $F_{1}$ (see [29]).

\section{Integral representations}

Here, we present various integral representations of the generalized Srivastava's triple hypergeometric functions (2.1), (2.2) and (2.3) some of which contains Laguerre polynomial, Whittaker function, Bessel function and modified Bessel function in the kernel. We begin by establishing an integral representation of the extended second Appell's hypergeometric function in (1.5), which is sure to be new and (potentially) useful in the following lemma.

Lemma 3.1. The following integral representation for $\mathrm{F}_{2}$ in (1.5) holds true:

$$
\begin{aligned}
F_{2}\left[(\alpha, p), \beta_{1}, \beta_{2} ; \gamma_{1}, \gamma_{2} ; x_{1}, x_{2}\right]= & \frac{1}{B\left(\beta_{1}, \gamma_{1}-\beta_{1}\right) B\left(\beta_{2}, \gamma_{2}-\beta_{2}\right)} \\
& \times \int_{0}^{1} \int_{0}^{1} t^{\beta_{1}-1} s^{\beta_{2}-1}(1-t)^{\gamma_{1}-\beta_{1}-1}(1-s)^{\gamma_{2}-\beta_{2}-1} \\
& \times{ }_{1} F_{0}\left[(\alpha, p) ;-; x_{1} t+x_{2} s\right] d t d s, \\
\left(\mathfrak{R}(p)>0 \quad \mathfrak{R}\left(\gamma_{j}\right)>\right. & \left.\Re\left(\beta_{j}\right)>0, \quad(j=1,2) \text { when } p=0\right),
\end{aligned}
$$

where $\mathrm{B}(\alpha, \beta)$ is the familiar Beta function defined by

$$
B(\alpha, \beta):= \begin{cases}\int_{0}^{1} t^{\alpha-1}(1-t)^{\beta-1} d t, & (\min \{\mathfrak{R}(\alpha), \mathfrak{R}(\beta)\}>0), \\ \frac{\Gamma(\alpha) \Gamma(\beta)}{\Gamma(\alpha+\beta)}, & \left(\alpha, \beta \in \mathbb{C} \backslash \mathbb{Z}_{0}^{-}\right) .\end{cases}
$$


Proof. Applying the following elementary identity

$$
\begin{gathered}
\frac{(\beta)_{v}}{(\gamma)_{v}}=\frac{B(\beta+v, \gamma-\beta)}{B(\beta, \gamma-\beta)}=\frac{1}{B(\beta, \gamma-\beta)} \int_{0}^{1} t^{\beta+v-1}(1-t)^{\gamma-\beta-1} d t, \\
(\mathfrak{R}(\gamma)>\mathfrak{R}(\beta)>\max \{0,-\mathfrak{R}(v)\}),
\end{gathered}
$$

to (1.5) and using Definition 1.1, we get the desired result (3.1).

Theorem 3.2. The following integral representation for $\mathrm{H}_{\mathrm{A}}$ in (2.1) holds true:

$$
\begin{aligned}
\mathrm{H}_{\mathrm{A}}\left[(\alpha, p), \beta_{1}, \beta_{2} ; \gamma_{1}, \gamma_{2} ; x_{1}, x_{2}, x_{3}\right]= & \frac{1}{\Gamma(\alpha) \Gamma\left(\beta_{1}\right)} \int_{0}^{\infty} \int_{0}^{\infty} e^{-s-\frac{p}{s}-t} t^{\alpha-1} s^{\beta_{1}-1} \\
& \times{ }_{0} \mathrm{~F}_{1}\left(-; \gamma_{1} ; x_{1} s t\right){ }_{1} \mathrm{~F}_{1}\left(\beta_{2} ; \gamma_{2} ; x_{2} s+x_{3} t\right) d t d s, \\
\left(\mathfrak{R}(p)>0, \quad \max \left\{\mathfrak{R}\left(x_{2}\right), \mathfrak{R}\left(x_{3}\right)\right\}<\right. & \left.1, \quad \min \left\{\mathfrak{R}(\alpha), \mathfrak{R}\left(\beta_{1}\right)\right\}>0 \text { when } p=0\right) .
\end{aligned}
$$

Proof. Using the integral representation (1.4) to $(\alpha ; p)_{l+n}$ and $\left(\beta_{1}\right)_{l+m}$ in (2.1) and using the elementary series identity [27, p. 52, Eq. 1.6(2)]:

$$
\sum_{m_{1}, m_{2}=0}^{\infty} \Omega\left(m_{1}+m_{2}\right) \frac{x_{1}^{m_{1}}}{m_{1} !} \frac{x_{2}^{m_{2}}}{m_{2} !}=\sum_{m=0}^{\infty} \Omega(m) \frac{\left(x_{1}+x_{2}\right)^{m}}{m !},
$$

we are led to the desired result.

Theorem 3.3. The following integral representation for $\mathrm{H}_{\mathrm{A}}$ in (2.1) holds true:

$$
\begin{aligned}
& \mathrm{H}_{\mathrm{A}}\left[(\alpha, p), \beta_{1}, \beta_{2} ; \gamma_{1}, \gamma_{2} ; x_{1}, x_{2}, x_{3}\right]= \frac{1}{\Gamma(\alpha) \Gamma\left(\beta_{1}\right) \Gamma\left(\beta_{2}\right)} \int_{0}^{\infty} \int_{0}^{\infty} \int_{0}^{\infty} e^{-s-\frac{p}{s}-t} t^{\alpha-1} s^{\beta_{1}-1} u^{\beta_{2}-1} \\
& \times{ }_{0} F_{1}\left(-; \gamma_{1} ; x_{1} s t\right){ }_{0} F_{1}\left(-; \gamma_{2} ; x_{2} u s+x_{3} u t\right) d t d s d u, \\
&\left(\mathfrak{R}(p)>0, \quad \min \left\{\mathfrak{R}(\alpha), \mathfrak{R}\left(\beta_{1}\right), \mathfrak{R}\left(\beta_{2}\right)\right\}>0 \text { when } p=0\right) .
\end{aligned}
$$

Proof. Using the elementary integral representation [13, p. 678, Eq.(4)]:

$$
{ }_{1} \mathrm{~F}_{1}(\lambda ; \mu ; z)=\frac{1}{\Gamma(\lambda)} \int_{0}^{\infty} \mathrm{t}^{\lambda-1} e^{-\mathrm{t}}{ }_{0} \mathrm{~F}_{1}(-; \mu ; z \mathrm{t}) \mathrm{dt}, \quad(\Re(\lambda)>0),
$$

in (3.2), we are led to the desired integral representation.

Theorem 3.4. The following integral representation for $\mathrm{H}_{\mathrm{B}}$ in (2.2) holds true:

$$
\begin{aligned}
& \mathrm{H}_{\mathrm{B}}\left[(\alpha, p), \beta_{1}, \beta_{2} ; \gamma_{1}, \gamma_{2}, \gamma_{3} ; x_{1}, x_{2}, x_{3}\right]= \frac{1}{\Gamma(\alpha) \Gamma\left(\beta_{1}\right)} \int_{0}^{\infty} \int_{0}^{\infty} e^{-s-\frac{p}{s}-t} t^{\alpha-1} s^{\beta_{1}-1} \\
& \times{ }_{0} F_{1}\left(-; \gamma_{1} ; x_{1} s t\right) \Psi_{2}\left(\beta_{2} ; \gamma_{2}, \gamma_{3} ; x_{2} s, x_{3} t\right) d t d s, \\
&\left(\mathfrak{R}(p)>0, \quad \mathfrak{R}\left(x_{2}\right)<1, \quad \mathfrak{R}\left(x_{3}\right)<1, \quad \mathfrak{R}(\alpha)>0, \quad \mathfrak{R}\left(\beta_{1}\right)>0 \text { when } p=0\right),
\end{aligned}
$$

where $\Psi_{2}$ is the confluent form of Appell series in two variables in (1.6).

Proof. Applying the integral representation (1.4) to $(\alpha ; p)_{l+n}$ and $\left(\beta_{1}\right)_{l+m}$ in (2.2) and using Definition 1.6 in the resulting identity, we are led to the desired result.

Theorem 3.5. The following triple integral representation for $\mathrm{H}_{\mathrm{B}}$ in (2.2) holds true:

$$
\begin{aligned}
& \mathrm{H}_{\mathrm{B}}\left[(\alpha, p), \beta_{1}, \beta_{2} ; \gamma_{1}, \gamma_{2}, \gamma_{3} ; x_{1}, x_{2}, x_{3}\right]= \frac{1}{\Gamma(\alpha) \Gamma\left(\beta_{1}\right) \Gamma\left(\beta_{2}\right)} \int_{0}^{\infty} \int_{0}^{\infty} \int_{0}^{\infty} e^{-s-\frac{p}{s}-t-u} t^{\alpha-1} s^{\beta_{1}-1} u^{\beta_{2}-1} \\
& \times{ }_{0} F_{1}\left(-; \gamma_{1} ; x_{1} s t\right){ }_{0} F_{1}\left(-; \gamma_{2} ; x_{2} u s\right){ }_{0} F_{1}\left(-; \gamma_{3} ; x_{3} u t\right) d t d s d u, \\
&\left(\mathfrak{R}(p)>0, \quad \min \left\{\mathfrak{R}(\alpha), \mathfrak{R}\left(\beta_{1}\right), \mathfrak{R}\left(\beta_{2}\right)\right\}>0 \text { when } p=0\right) .
\end{aligned}
$$


Proof. Applying the integral representation (1.4) to $(\alpha ; p)_{l+n},\left(\beta_{1}\right)_{l+m}$ and $\left(\beta_{2}\right)_{m+n}$ in (2.2) and using Definition 1.1 (with $p=0$ ) in the resulting identity, we are led to the desired result.

Theorem 3.6. The following triple integral representation for $\mathrm{H}_{\mathrm{B}}$ in (2.2) holds true:

$$
\begin{aligned}
H_{B}\left[(\alpha, p), \beta_{1}, \beta_{2} ; \gamma_{1}, \gamma_{2}, \gamma_{3} ; x_{1}, x_{2}, x_{3}\right]= & \frac{1}{B\left(\beta_{1}, \gamma_{1}-\beta_{1}\right) B\left(\beta_{2}, \gamma_{3}-\beta_{2}\right) B\left(1-\gamma_{1}+\beta_{1}, \gamma_{1}+\gamma_{2}-\beta_{1}-1\right)} \\
& \times \int_{0}^{1} \int_{0}^{1} \int_{0}^{1} u^{\beta_{1}-1} v^{\beta_{1}-\gamma_{1}} w^{\beta_{2}-1}(1-u)^{\gamma_{1}-\gamma_{3}-\beta_{1}+\beta_{2}}(1-v)^{\gamma_{1}+\gamma_{2}-\beta_{1}-2} \\
& \times{ }_{1} F_{0}\left[(\alpha, p) ;-; u x_{1}+w x_{3}\right] \\
& \times\left(1-u-w+u w-u v w x_{2}\right)^{\gamma_{3}-\beta_{2}-1} d u d v d w,
\end{aligned}
$$

$\left(\mathfrak{R}(\mathrm{p})>0, \quad 0<\mathfrak{R}\left(\gamma_{1}-\beta_{1}\right)<1, \quad \mathfrak{R}\left(\beta_{1}\right)>1, \quad \mathfrak{R}\left(\gamma_{1}+\gamma_{2}-\beta_{1}\right)>1, \quad \mathfrak{R}\left(\gamma_{3}\right)>\mathfrak{R}\left(\beta_{2}\right)>0\right.$ when $\left.p=0\right)$.

Proof. We find from (2.2) that

$$
H_{B}\left[(\alpha, p), \beta_{1}, \beta_{2} ; \gamma_{1}, \gamma_{2}, \gamma_{3} ; x_{1}, x_{2}\right]=\sum_{n=0}^{\infty} \frac{\left(\beta_{1}\right)_{n}\left(\beta_{2}\right)_{n}}{\left(\gamma_{2}\right)_{n}} F_{2}\left[(\alpha, p), \beta_{1}+n, \beta_{2}+n ; \gamma_{1}, \gamma_{3} ; x_{1}, x_{3}\right] \frac{x_{2}^{n}}{n !} .
$$

Employing the integral representation (3.1) in (3.6), changing the order of summation and integration, which is verified by the uniform convergence of the involved series, we obtain

$$
\begin{aligned}
& H_{B}\left[(\alpha, p), \beta_{1}, \beta_{2} ; \gamma_{1}, \gamma_{2}, \gamma_{3} ; x_{1}, x_{2}, x_{3}\right]=\frac{1}{B\left(\beta_{1}, \gamma_{1}-\beta_{1}\right) B\left(\beta_{2}, \gamma_{3}-\beta_{2}\right)} \\
& \times \int_{0}^{1} \int_{0}^{1} u^{\beta_{1}-1} w^{\beta_{2}-1}(1-u)^{\gamma_{1}-\beta_{1}-1}{ }_{1} F_{0}\left[(\alpha, p) ;-; u x_{1}+w x_{3}\right]
\end{aligned}
$$

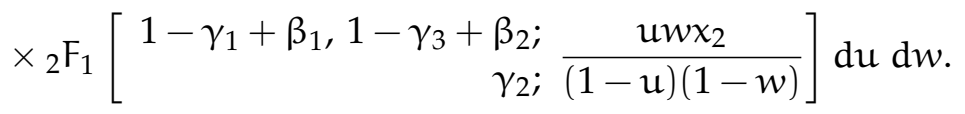

Using the following integral representation (see [6, p.59, Eq. (10)], [13] and [25, Chapter 1]):

$$
\begin{gathered}
{ }_{2} \mathrm{~F}_{1}(\mathrm{a}, \mathrm{b} ; \mathrm{c} ; z)=\frac{\Gamma(\mathrm{c})}{\Gamma(\mathrm{a}) \Gamma(\mathrm{c}-\mathrm{a})} \int_{0}^{1} \mathrm{t}^{\mathrm{a}-1}(1-\mathrm{t})^{\mathrm{c}-\mathrm{a}-1}(1-z \mathrm{t})^{-\mathrm{b}} \mathrm{dt}, \\
(\mathfrak{R}(\mathrm{c})>\mathfrak{R}(\mathrm{a})>0, \quad|\arg (1-z)| \leqslant \pi-\epsilon \quad(0<\epsilon<\pi)),
\end{gathered}
$$

in (3.7), we are led to the desired integral representation (3.5).

Theorem 3.7. The following integral representation for $\mathrm{H}_{\mathrm{C}}$ in (2.3) holds true:

$$
\begin{aligned}
& \mathrm{H}_{\mathrm{C}}\left[(\alpha, p), \beta_{1}, \beta_{2} ; \gamma ; x_{1}, x_{2}, x_{3}\right]= \frac{1}{\Gamma(\alpha) \Gamma\left(\beta_{1}\right)} \\
& \times \int_{0}^{\infty} \int_{0}^{\infty} e^{-s-\frac{p}{s}-t} t^{\alpha-1} s^{\beta_{1}-1} \Phi_{3}\left(\beta_{2} ; \gamma ; x_{2} s+x_{3} t, x_{1} s t\right) d t d s, \\
&\left(\mathfrak{R}(p)>0, \quad \mathfrak{R}\left(x_{2}\right)<1, \quad \mathfrak{R}\left(x_{3}\right)<1, \quad \mathfrak{R}(\alpha)>0, \quad \mathfrak{R}\left(\beta_{1}\right)>0 \text { when } p=0\right),
\end{aligned}
$$

where $\Phi_{2}$ is the confluent form of Appell series in two variables in (1.7).

Proof. Applying the integral representation (1.4) to $(\alpha ; p)_{l+n}$ and $\left(\beta_{1}\right)_{l+m}$ in (2.3) and using Definition 1.7 in the resulting identity, we are led to the desired result. 
Theorem 3.8. The following triple integral representation for $\mathrm{H}_{\mathrm{C}}$ in (2.3) holds true:

$$
\begin{aligned}
& H_{C}\left[(\alpha, p), \beta_{1}, \beta_{2} ; \gamma ; x_{1}, x_{2}, x_{3}\right]= \frac{1}{\Gamma(\alpha) \Gamma\left(\beta_{1}\right) \Gamma\left(\beta_{2}\right)} \int_{0}^{\infty} \int_{0}^{\infty} \int_{0}^{\infty} e^{-s-\frac{p}{s}-t-u} t^{\alpha-1} s^{\beta_{1}-1} u^{\beta_{2}-1} \\
& \times{ }_{0} F_{1}\left(-; \gamma ; x_{1} s t+x_{2} u s+x_{3} u t\right) d t d s d u, \\
&\left(\Re(p)>0, \quad \min \left\{\mathfrak{R}(\alpha), \mathfrak{R}\left(\beta_{1}\right), \mathfrak{R}\left(\beta_{2}\right)\right\}>0 \text { when } p=0\right) .
\end{aligned}
$$

Proof. Applying the integral representation (1.4) to $(\alpha ; p)_{l+n},\left(\beta_{1}\right)_{l+m}$, and $\left(\beta_{2}\right)_{m+n}$ in (2.3) and using Definition 1.1 in the resulting identity, we are led to the desired result.

The Laguerre polynomial $\mathrm{L}_{n}^{(\alpha)}(x)$ (see, e.g., [13]) of order (index) $\alpha$ and degree $n$ in $x$, Whittaker function of two variables $M_{k, m, n}(x, y)$ (see [20, p. 100]), Bessel function $J_{v}(z)$ and the modified Bessel function $\mathrm{I}_{v}(z)$ (see, e.g., [13]; see also [7-9]) are expressible in terms of the confluent function $\Psi_{2}$ and the confluent hypergeometric functions ${ }_{0} \mathrm{~F}_{1}$, respectively, as follows:

$$
\begin{aligned}
\mathrm{L}_{\mathrm{n}}^{(\alpha)}(x) & =\frac{(\alpha+1)_{n}}{\mathrm{n} !}{ }_{1} \mathrm{~F}_{1}(-n ; \alpha+1 ; x), \\
\mathrm{M}_{\mathrm{k}, \mathrm{m}, \mathrm{n}}(x, y) & =x^{\mathrm{m}+\frac{1}{2}} y^{\mathrm{n}+\frac{1}{2}} e^{-\frac{1}{2}(x+y)} \Psi_{2}(\mathrm{~m}+\mathrm{n}-\mathrm{k}+1 ; 2 \mathrm{~m}+1,2 \mathrm{n}+1 ; x, y), \\
J_{v}(z) & =\frac{\left(\frac{z}{2}\right)^{v}}{\Gamma(v+1)}{ }_{0} \mathrm{~F}_{1}\left(-; v+1 ;-\frac{1}{4} z^{2}\right), \quad\left(v \in \mathbb{C} \backslash \mathbb{Z}^{-}\right),
\end{aligned}
$$

and

$$
\mathrm{I}_{v}(z)=\frac{\left(\frac{z}{2}\right)^{v}}{\Gamma(v+1)}{ }_{0} \mathrm{~F}_{1}\left(-; v+1 ; \frac{1}{4} z^{2}\right), \quad\left(v \in \mathbb{C} \backslash \mathbb{Z}^{-}\right) .
$$

Now, applying the relationships (3.9) to (3.2), (3.11) and (3.12) to (3.2), and (3.9), (3.11) and (3.12) to (3.2), respectively, we can deduce certain interesting integral representations for the generalized Srivastava's triple hypergeometric function $H_{A}$ in (2.1) asserted by Corollaries 3.9, 3.10, and 3.11. Their proofs are omitted.

Corollary 3.9. The following integral representation for $\mathrm{H}_{\mathrm{A}}$ in (2.1) holds true:

$$
\begin{aligned}
H_{A}\left[(\alpha, x), \beta_{1},-m ; \gamma_{1}, \gamma_{2}+1 ; x_{1}, x_{2}, x_{3}\right]= & \frac{m !}{\left(\gamma_{2}+1\right) m \Gamma(\alpha) \Gamma\left(\beta_{1}\right)} \\
& \times \int_{0}^{\infty} \int_{0}^{\infty} e^{-s-\frac{p}{s}-t} t^{\alpha-1} s^{\beta_{1}-1}{ }_{0} F_{1}\left(-; \gamma_{1} ; x_{1} s t\right) L_{m}^{\left(\gamma_{2}\right)}\left(x_{2} s+x_{3} t\right) d t d s,
\end{aligned}
$$

provided that the involved integral is convergent.

Corollary 3.10. Each of the following double integral representations holds true:

$$
\begin{aligned}
\mathrm{H}_{\mathrm{A}}\left[(\alpha, p), \beta_{1}, \beta_{2} ; \gamma_{1}+1, \gamma_{2} ;-\chi_{1}, x_{2}, x_{3}\right]= & \frac{\Gamma\left(\gamma_{1}+1\right) x_{1}^{-\frac{\gamma_{1}}{2}}}{\Gamma(\alpha) \Gamma\left(\beta_{1}\right)} \times \int_{0}^{\infty} \int_{0}^{\infty} e^{-s-\frac{p}{s}-t} t^{\alpha-\frac{\gamma_{1}}{2}-1} s^{\beta_{1}-\frac{\gamma_{1}}{2}-1} \\
& \times J_{\gamma_{1}}\left(2 \sqrt{x_{1} s t}\right){ }_{1} F_{1}\left(\beta_{2} ; \gamma_{2} ; x_{2} s+x_{3} t\right) d t d s
\end{aligned}
$$

and

$$
\begin{aligned}
H_{A}\left[(\alpha, p), \beta_{1}, \beta_{2} ; \gamma_{1}+1, \gamma_{2} ; x_{1}, x_{2}, x_{3}\right]= & \frac{\Gamma\left(\gamma_{1}+1\right) x_{1}^{-\frac{\gamma_{1}}{2}}}{\Gamma(\alpha) \Gamma\left(\beta_{1}\right)} \times \int_{0}^{\infty} \int_{0}^{\infty} e^{-s-\frac{p}{s}-t} t^{\alpha-\frac{\gamma_{1}}{2}-1} s^{\beta_{1}-\frac{\gamma_{1}}{2}-1} \\
& \times I_{\gamma_{1}}\left(2 \sqrt{x_{1} s t}\right){ }_{1} F_{1}\left(\beta_{2} ; \gamma_{2} ; x_{2} s+x_{3} t\right) d t d s,
\end{aligned}
$$

provided that the involved integrals are convergent. 
Corollary 3.11. Each of the following double integral representations holds true:

$$
\begin{aligned}
H_{A}\left[(\alpha, p), \beta_{1},-m ; \gamma_{1}+1, \gamma_{2}+1 ;-x_{1}, x_{2}, x_{3}\right]= & \frac{m ! \Gamma\left(\gamma_{1}+1\right) x_{1}^{-\frac{\gamma_{1}}{2}}}{\left(\gamma_{2}+1\right)_{m} \Gamma(\alpha) \Gamma\left(\beta_{1}\right)} \times \int_{0}^{\infty} \int_{0}^{\infty} e^{-s-\frac{p}{s}-t} t^{\alpha-\frac{\gamma_{1}}{2}-1} s^{\beta_{1}-\frac{\gamma_{1}}{2}-1} \\
& \times J_{\gamma_{1}}\left(2 \sqrt{x_{1} s t}\right) L_{m}^{\left(\gamma_{2}\right)}\left(x_{2} s+x_{3} t\right) d t d s
\end{aligned}
$$

and

$$
\begin{aligned}
\mathrm{H}_{A}\left[(\alpha, p), \beta_{1},-\mathrm{m} ; \gamma_{1}+1, \gamma_{2}+1 ; x_{1}, x_{2}, x_{3}\right]= & \frac{\mathrm{m} ! \Gamma\left(\gamma_{1}+1\right) x_{1}^{-\frac{\gamma_{1}}{2}}}{\left(\gamma_{2}+1\right)_{m} \Gamma(\alpha) \Gamma\left(\beta_{1}\right)} \times \int_{0}^{\infty} \int_{0}^{\infty} e^{-s-\frac{p}{s}-t} t^{\alpha-\frac{\gamma_{1}}{2}-1} s^{\beta_{1}-\frac{\gamma_{1}}{2}-1} \\
& \times I_{\gamma_{1}}\left(2 \sqrt{x_{1} s t}\right) L_{m}^{\left(\gamma_{2}\right)}\left(x_{2} s+x_{3} t\right) d t d s,
\end{aligned}
$$

provided that the involved integrals are convergent.

Further, by applying (3.10) to (3.3), (3.11) and (3.12) to (3.3) and (3.10), (3.11) and (3.12) to (3.3), (3.11) and (3.12) to (3.4) respectively, we can deduce certain integral representations for the generalized Srivastava's triple hypergeometric function $\mathrm{H}_{\mathrm{B}}$ in (2.2), which are asserted by Corollaries 3.12, 3.13, 3.14 and 3.15. We omit the detailed account involving proof for each formula.

Corollary 3.12. The following integral representation for $\mathrm{H}_{\mathrm{B}}$ in (2.2) holds true:

$$
\begin{aligned}
\mathrm{H}_{\mathrm{B}}[ & \left.(\lambda, p), \mu, \sigma+\rho-\mathrm{k}+1 ; v, 2 \sigma+1,2 \rho+1 ; x_{1}, x_{2}, x_{3}\right] \\
= & \frac{x_{2}^{-\sigma-\frac{1}{2}} x_{3}^{-\rho-\frac{1}{2}}}{\Gamma(\lambda) \Gamma(\mu)} \times \int_{0}^{\infty} \int_{0}^{\infty} e^{-\left(1-\frac{1}{2} x_{2}\right) s-\frac{p}{s}-\left(1-\frac{1}{2} x_{3}\right) t} t^{\lambda-\rho-\frac{3}{2}} s^{\mu-\sigma-\frac{3}{2}} \\
\quad & \times{ }_{0} F_{1}\left(-; v ; x_{1} s t\right) M_{k, \sigma, \rho}\left(x_{2} s, x_{3} t\right) d t d s,
\end{aligned}
$$

provided that the involved integral is convergent.

Corollary 3.13. Each of the following double integral representations holds true:

$$
\begin{aligned}
H_{B}\left[(\alpha, p), \beta_{1}, \beta_{2} ; \gamma_{1}+1, \gamma_{2}, \gamma_{3} ;-x_{1}, x_{2}, x_{3}\right]= & \frac{\Gamma\left(\gamma_{1}+1\right) x_{1}^{-\frac{\gamma_{1}}{2}}}{\Gamma(\alpha) \Gamma\left(\beta_{1}\right)} \times \int_{0}^{\infty} \int_{0}^{\infty} e^{-s-\frac{p}{s}-t} t^{\alpha-\frac{\gamma_{1}}{2}-1} s^{\beta_{1}-\frac{\gamma_{1}}{2}-1} \\
& \times J_{\gamma_{1}}\left(2 \sqrt{\chi_{1} s t}\right) \Psi_{2}\left(\beta_{2} ; \gamma_{2}, \gamma_{3} ; x_{2} s, x_{3} t\right) d t d s,
\end{aligned}
$$

and

$$
\begin{aligned}
H_{B}\left[(\alpha, p), \beta_{1}, \beta_{2} ; \gamma_{1}+1, \gamma_{2}, \gamma_{3} ; x_{1}, x_{2}, x_{3}\right]= & \frac{\Gamma\left(\gamma_{1}+1\right) x_{1}^{-\frac{\gamma_{1}}{2}}}{\Gamma(\alpha) \Gamma\left(\beta_{1}\right)} \times \int_{0}^{\infty} \int_{0}^{\infty} e^{-s-\frac{p}{s}-t} t^{\alpha-\frac{\gamma_{1}}{2}-1} s^{\beta_{1}-\frac{\gamma_{1}}{2}-1} \\
& \left.\times I_{\gamma_{1}}\left(2 \sqrt{x_{1} s t}\right)\right) \Psi_{2}\left(\beta_{2} ; \gamma_{2}, \gamma_{3} ; x_{2} s, x_{3} t\right) d t d s
\end{aligned}
$$

provided that the involved integrals are convergent.

Corollary 3.14. Each of the following double integral representations holds true:

$$
\begin{aligned}
\mathrm{H}_{\mathrm{B}}\left[(\lambda, p), \mu, \sigma+\rho-\mathrm{k}+1 ; v+1,2 \sigma+1,2 \rho+1 ;-x_{1}, x_{2}, x_{3}\right] \\
=\frac{\Gamma(v+1) x_{1}^{-\frac{1}{2} v} x_{2}^{-\sigma-\frac{1}{2}} x_{3}^{-\rho-\frac{1}{2}}}{\Gamma(\lambda) \Gamma(\mu)} \\
\quad \times \int_{0}^{\infty} \int_{0}^{\infty} e^{-\left(1-\frac{1}{2} x_{2}\right) s-\frac{p}{s}-\left(1-\frac{1}{2} x_{3}\right) t} t^{\lambda-\rho-\frac{1}{2} v-\frac{3}{2}} \\
\quad \times s^{\mu-\sigma-\frac{1}{2} v-\frac{3}{2}} J_{v}\left(2 \sqrt{\chi_{1} s t}\right) M_{k, \sigma, \rho}\left(x_{2} s, x_{3} t\right) d t d s,
\end{aligned}
$$


and

$$
\begin{aligned}
\mathrm{H}_{\mathrm{B}}\left[(\lambda, p), \mu, \sigma+\rho-\mathrm{k}+1 ; v+1,2 \sigma+1,2 \rho+1 ; x_{1}, x_{2}, x_{3}\right] \\
=\frac{\Gamma(v+1) x_{1}^{-\frac{1}{2} v} x_{2}^{-\sigma-\frac{1}{2}} x_{3}^{-\rho-\frac{1}{2}}}{\Gamma(\lambda) \Gamma(\mu)} \\
\quad \times \int_{0}^{\infty} \int_{0}^{\infty} e^{-\left(1-\frac{1}{2} x_{2}\right) s-\frac{p}{s}-\left(1-\frac{1}{2} x_{3}\right) t} t^{\lambda-\rho-\frac{1}{2} v-\frac{3}{2}} \\
\quad \times s^{\mu-\sigma-\frac{1}{2} v-\frac{3}{2}} I_{v}\left(2 \sqrt{\chi_{1} s t}\right) M_{k, \sigma, \rho}\left(x_{2} s, x_{3} t\right) d t d s,
\end{aligned}
$$

provided that the involved integrals are convergent.

Corollary 3.15. Each of the following double integral representations holds true:

$$
\begin{aligned}
\mathrm{H}_{\mathrm{B}}\left[(\alpha, p), \beta_{1}, \beta_{2} ; \gamma_{1}+1, \gamma_{2}+1, \gamma_{3}+1 ;-x_{1},-x_{2},-x_{3}\right] \\
=\frac{\Gamma\left(\gamma_{1}+1\right) \Gamma\left(\gamma_{2}+1\right) \Gamma\left(\gamma_{3}+1\right) x_{1}^{\frac{-\gamma_{1}}{2}} x_{2}^{\frac{-\gamma_{2}}{2}} x_{3}^{\frac{-\gamma_{3}}{2}}}{\Gamma(\alpha) \Gamma\left(\beta_{1}\right) \Gamma\left(\beta_{2}\right)} \\
\quad \times \int_{0}^{\infty} \int_{0}^{\infty} \int_{0}^{\infty} e^{-s-\frac{p}{s}-t-u} t^{\alpha-\frac{\gamma_{1}}{2}-\frac{\gamma_{3}}{2}-1} s^{\beta_{1}-\frac{\gamma_{1}}{2}-\frac{\gamma_{2}}{2}-1} u^{\beta_{2}-\frac{\gamma_{2}}{2}-\frac{\gamma_{3}}{2}-1} \\
\quad \times J_{\gamma_{1}}\left(2 \sqrt{x_{1} s t}\right) J_{\gamma_{2}}\left(2 \sqrt{x_{2} u s}\right) J_{\gamma_{3}}\left(2 \sqrt{x_{3} u t}\right) d t d s d u,
\end{aligned}
$$

and

$$
\begin{aligned}
H_{B}\left[(\alpha, p), \beta_{1}, \beta_{2} ; \gamma_{1}+1, \gamma_{2}+1, \gamma_{3}+1 ; x_{1}, x_{2}, x_{3}\right] \\
=\frac{\Gamma\left(\gamma_{1}+1\right) \Gamma\left(\gamma_{2}+1\right) \Gamma\left(\gamma_{3}+1\right) x_{1}^{\frac{-\gamma_{1}}{2}} x_{2}^{\frac{-\gamma_{2}}{2}} x_{3}^{\frac{-\gamma_{3}}{2}}}{\Gamma(\alpha) \Gamma\left(\beta_{1}\right) \Gamma\left(\beta_{2}\right)} \\
\quad \times \int_{0}^{\infty} \int_{0}^{\infty} \int_{0}^{\infty} e^{-s-\frac{p}{s}-t-u} t^{\alpha-\frac{\gamma_{1}}{2}-\frac{\gamma_{3}}{2}-1} s^{\beta_{1}-\frac{\gamma_{1}}{2}-\frac{\gamma_{2}}{2}-1} u^{\beta_{2}-\frac{\gamma_{2}}{2}-\frac{\gamma_{3}}{2}-1} \\
\quad \times \mathrm{I}_{\gamma_{1}}\left(2 \sqrt{\chi_{1} s t}\right) \mathrm{I}_{\gamma_{2}}\left(2 \sqrt{\chi_{2} u s}\right) \mathrm{I}_{\gamma_{3}}\left(2 \sqrt{\chi_{3} u t}\right) d t d s d u,
\end{aligned}
$$

provided that the involved integrals are convergent.

\section{Derivative and recurrence formulas}

Here, we derive derivative formulas and recurrence formulas for the generalized Srivastava's triple hypergeometric functions $H_{A}, H_{B}$ and $H_{C}$ in (2.1), (2.2) and (2.3).

\section{Derivative formulas}

Differentiating, partially, both sides of (2.1), (2.2) and (2.3) with respect to the variables $x_{1}, x_{2}$, and $x_{3}$, $l, m$ and $n$ times, respectively, we obtain the derivative formulas for the functions $H_{A}, H_{B}$ and $H_{C}$ in (2.1), (2.2) and (2.3), which are given in Theorem 4.1.

Theorem 4.1. The following derivative formulas for $\mathrm{H}_{A}, \mathrm{H}_{B}$ and $\mathrm{H}_{C}$ hold true:

$$
\begin{aligned}
& \frac{\partial^{l+m+n}}{\partial x_{1}^{l} \partial x_{2}^{m} \partial x_{3}^{n}} H_{A}\left[(\alpha, p), \beta_{1}, \beta_{2} ; \gamma_{1}, \gamma_{2} ; x_{1}, x_{2}, x_{3}\right] \\
& \quad=\frac{(\alpha)_{l+n}\left(\beta_{1}\right)_{l+m}\left(\beta_{2}\right)_{m+n}}{\left(\gamma_{1}\right)_{l}\left(\gamma_{2}\right)_{m+n}} \\
& \quad \times H_{A}\left[(\alpha+l+n, p), \beta_{1}+l+m, \beta_{2}+m+n ; \gamma_{1}+l, \gamma_{2}+m+n ; x_{1}, x_{2}, x_{3}\right],
\end{aligned}
$$




$$
\begin{aligned}
& \frac{\partial^{l+m+n}}{\partial x_{1}^{l} \partial x_{2}^{m} \partial x_{3}^{n}} H_{B}\left[(\alpha, p), \beta_{1}, \beta_{2} ; \gamma_{1}, \gamma_{2}, \gamma_{3} ; x_{1}, x_{2}, x_{3}\right] \\
& =\frac{(\alpha)_{l+n}\left(\beta_{1}\right)_{l+m}\left(\beta_{2}\right)_{m+n}}{\left(\gamma_{1}\right)_{l}\left(\gamma_{2}\right)_{m}\left(\gamma_{3}\right)_{n}} \\
& \quad \times H_{B}\left[(\alpha+l+n, p), \beta_{1}+l+m, \beta_{2}+m+n ; \gamma_{1}+l, \gamma_{2}+m, \gamma_{3}+n ; x_{1}, x_{2}, x_{3}\right], \\
& \frac{\partial^{l+m+n}}{\partial x_{1}^{l} \partial x_{2}^{m} \partial x_{3}^{n}} H_{C}\left[(\alpha, p), \beta_{1}, \beta_{2} ; \gamma ; x_{1}, x_{2}, x_{3}\right] \\
& =\frac{(\alpha)_{l+n}\left(\beta_{1}\right)_{l+m}\left(\beta_{2}\right)_{m+n}}{(\gamma)_{l+m+n}} \\
& \quad \times H_{C}\left[(\alpha+l+n, p), \beta_{1}+l+m, \beta_{2}+m+n ; \gamma+l+m+n ; x_{1}, x_{2}, x_{3}\right] .
\end{aligned}
$$

\section{Recurrence relations}

We present recurrence relations for the functions $H_{A}, H_{B}$ and $H_{C}$ in (2.1), (2.2) and (2.3), which are given in Theorems 4.2-4.5.

Theorem 4.2. The following recurrence relation for $\mathrm{H}_{\mathrm{A}}$ holds true:

$$
\begin{aligned}
H_{A}\left[(\alpha, p), \beta_{1}, \beta_{2} ; \gamma_{1}, \gamma_{2} ; x_{1}, x_{2}, x_{3}\right]= & H_{A}\left[(\alpha, p), \beta_{1}, \beta_{2} ; \gamma_{1}-1, \gamma_{2} ; x_{1}, x_{2}, x_{3}\right] \\
& +\frac{\alpha \beta_{1} x_{1}}{\gamma_{1}\left(1-\gamma_{1}\right)} \\
& \times H_{A}\left[(\alpha+1, p), \beta_{1}+1, \beta_{2} ; \gamma_{1}+1, \gamma_{2} ; x_{1}, x_{2}, x_{3}\right] .
\end{aligned}
$$

Proof. Using the well-known contiguous relation for the function ${ }_{0} \mathrm{~F}_{1}$ (see [14, p. 12]):

$$
{ }_{0} \mathrm{~F}_{1}(-; \gamma-1 ; x)-{ }_{0} \mathrm{~F}_{1}(-; \gamma ; x)-\frac{x}{\gamma(\gamma-1)}{ }_{0} \mathrm{~F}_{1}(-; \gamma+1 ; x)=0,
$$

in the integral representation (3.2), we are led to the desired result.

Theorem 4.3. The following recurrence relation for $\mathrm{H}_{\mathrm{A}}$ holds true:

$$
\begin{aligned}
\left(\gamma_{2}-\beta_{2}-1\right) H_{A}\left[(\alpha, p), \beta_{1}, \beta_{2} ; \gamma_{1}, \gamma_{2} ; x_{1}, x_{2}, x_{3}\right]= & \left(\gamma_{2}-1\right) H_{A}\left[(\alpha, p), \beta_{1}, \beta_{2} ; \gamma_{1}, \gamma_{2}-1 ; x_{1}, x_{2}, x_{3}\right] \\
& +\beta_{2} H_{A}\left[(\alpha, p), \beta_{1}, \beta_{2}+1 ; \gamma_{1}, \gamma_{2} ; x_{1}, x_{2}, x_{3}\right] .
\end{aligned}
$$

Proof. Using the well-known contiguous relation for the function ${ }_{1} \mathrm{~F}_{1}$ (see [13, p. 124, Eq.(6)]):

$$
(c-b-1){ }_{1} F_{1}(b ; c ; x)=(c-1){ }_{1} F_{1}(b ; c-1 ; x)-b{ }_{1} F_{1}(b+1 ; c ; x),
$$

in the integral representation (3.2), we are led to the desired result.

Theorem 4.4. The following recurrence relation for $\mathrm{H}_{\mathrm{B}}$ holds true:

$$
\begin{aligned}
H_{\mathrm{B}}\left[(\alpha, p), \beta_{1}, \beta_{2} ; \gamma_{1}, \gamma_{2}, \gamma_{3} ; x_{1}, x_{2}, x_{3}\right]= & \mathrm{H}_{\mathrm{B}}\left[(\alpha, p), \beta_{1}, \beta_{2} ; \gamma_{1}-1, \gamma_{2}, \gamma_{3} ; x_{1}, x_{2}, x_{3}\right] \\
& +\frac{\alpha \beta_{1} x_{1}}{\gamma(1-\gamma)} \\
& \times \mathrm{H}_{\mathrm{B}}\left[(\alpha+1, p), \beta_{1}+1, \beta_{2} ; \gamma_{1}+1, \gamma_{2}, \gamma_{3} ; x_{1}, x_{2}, x_{3}\right]
\end{aligned}
$$

Proof. A similar argument as in Theorem 4.2 together with the integral representation (3.3) will establish the desired result. 
Theorem 4.5. The following recurrence relation for $\mathrm{H}_{\mathrm{C}}$ holds true:

$$
\begin{aligned}
H_{C}\left[(\alpha, p), \beta_{1}, \beta_{2} ; \gamma ; x_{1}, x_{2}, x_{3}\right]= & H_{C}\left[(\alpha, p), \beta_{1}, \beta_{2} ; \gamma-1 ; x_{1}, x_{2}, x_{3}\right] \\
& +\frac{\alpha \beta_{1} x_{1}}{\gamma(1-\gamma)} H_{C}\left[(\alpha+1, p), \beta_{1}+1, \beta_{2} ; \gamma+1 ; x_{1}, x_{2}, x_{3}\right] \\
& +\frac{\beta_{1} \beta_{2} x_{2}}{\gamma(1-\gamma)} H_{C}\left[(\alpha, p), \beta_{1}+1, \beta_{2}+1 ; \gamma+1 ; x_{1}, x_{2}, x_{3}\right] \\
& +\frac{\alpha \beta_{2} x_{3}}{\gamma(1-\gamma)} H_{C}\left[(\alpha+1, p), \beta_{1}, \beta_{2}+1 ; \gamma+1 ; x_{1}, x_{2}, x_{3}\right] .
\end{aligned}
$$

Proof. By employing a similar argument as in Theorem 4.2 together with the integral representation (3.8), we are led to the desired result.

\section{Concluding remarks}

In our present investigation, by using the generalized Pochhammer symbol $(\lambda ; p)_{v}$ in (1.2), we have introduced the generalized Srivastava triple hypergeometric functions $H_{A}, H_{B}$ and $H_{C}$ in (2.1), (2.2) and (2.3), respectively, whose special cases when $x_{2}=0$ reduces to the extended Appell function $F_{2}$ and $F_{1}$ of two variables (see, e.g., [29]) and investigated their diverse properties such mainly as various integral representations, derivative formulas and recurrence relations. The special cases of the results presented here when $p=0$ would reduce to the corresponding well-known results for the Srivastava's triple hypergeometric functions (see, for details, [16-20, 26, 27]).

\section{References}

[1] M. A. Chaudhry, S. M. Zubair, Generalized incomplete gamma functions with applications, J. Comput. Appl. Math., 55 (1994), 99-124. 1

[2] M. A. Chaudhry, S. M. Zubair, On a class of incomplete gamma functions with applications, Chapman \& Hall/CRC, Boca Raton, FL, (2002). 1

[3] J.-S. Choi, R. K. Parmar, P. Chopra, Extended Mittag-Leffler function and associated fractional calculus operators, Georgian Math. J., (2017), (In press). 1

[4] J.-S. Choi, R. K. Parmar, T. K. Pogány, Mathieu-type series built by (p, q)-extended Gaussian hypergeometric function, ArXiv, 2016 (2016), 9 pages.

[5] J.-S. Choi, A. K. Rathie, R. K. Parmar, Extension of extended beta, hypergeometric and confluent hypergeometric functions, Honam Math. J., 36 (2014), 357-385. 1

[6] A. Erdélyi, W. Magnus, F. Oberhettinger, F. G. Tricomi, Higher transcendental functions, Vols. I, II, Based, in part, on notes left by Harry Bateman. McGraw-Hill Book Company, Inc., New York-Toronto-London, (1953). 3

[7] Y. L. Luke, Mathematical functions and their approximations, Academic Press, Inc. [Harcourt Brace Jovanovich, Publishers], New York-London, (1975). 3

[8] W. Magnus, F. Oberhettinger, R. P. Soni, Formulas and theorems for the special functions of mathematical physics, Third enlarged edition, Die Grundlehren der mathematischen Wissenschaften, Band 52 Springer-Verlag New York, Inc., New York, (1966).

[9] F. W. J. Olver, D. W. Lozier, R. F. Boisvert, C. W. Clark (Eds.), NIST handbook of mathematical functions [With 1 CD-ROM (Windows, Macintosh and UNIX)], US Department of Commerce, National Institute of Standards and Technology, Washington, DC, (2010); Cambridge University Press, Cambridge, London and New York, (2010). 3

[10] R. K. Parmar, Extended t-hypergeometric functions and associated properties, C. R. Math. Acad. Sci. Paris, 353 (2015), 421-426. 1

[11] R. K. Parmar, T. K. Pogány, Extended Srivastava's triple hypergeometric $\mathrm{H}_{\mathrm{A}, \mathrm{p}, \mathrm{q}}$ function and related bounding inequalities, J. Contemp. Math. Anal., (2016), (In press). 1

[12] T. K. Pogány, R. K. Parmar, On p-extended Mathieu series, ArXiv, 2016 (2016), 8 pages. 1

[13] E. D. Rainville, Special functions, Reprint of 1960 first edition, Chelsea Publishing Co., Bronx, N.Y., (1971). 1, 3, 3, 3,4

[14] L. J. Slater, Confluent hypergeometric functions, Cambridge University Press, New York, (1960). 4

[15] L. J. Slater, Generalized hypergeometric functions, Cambridge University Press, Cambridge, (1966). 1

[16] H. M. Srivastava, Hypergeometric functions of three variables, Gad̆ot nita ,15 (1964), 97-108. 2.1, 5

[17] H. M. Srivastava, On transformations of certain hypergeometric functions of three variables, Publ. Math. Debrecen, 12 (1965), 65-74. 
[18] H. M. Srivastava, On the reducibility of certain hypergeometric functions, Univ. Nac. Tucumn Rev. Ser. A, 16 (1966), 7-14.

[19] H.M. Srivastava, Relations between functions contiguous to certain hypergeometric functions of three variables, Proc. Nat. Acad. Sci. India Sect. A, 36 (1966), 377-385.

[20] H. M. Srivastava, Some integrals representing triple hypergeometric functions, Rend. Circ. Mat. Palermo, 16 (1967), 99-115. 2.1, 3, 5

[21] R. Srivastava, Some generalizations of Pochhammer's symbol and their associated families of hypergeometric functions and hypergeometric polynomials, Appl. Math. Inf. Sci., 7 (2013), 2195-2206. 1

[22] R. Srivastava, Some classes of generating functions associated with a certain family of extended and generalized hypergeometric functions, Appl. Math. Comput., 243 (2014), 132-137.

[23] H. M. Srivastava, A. Çetinkaya, İ. Onur Kiymaz, A certain generalized Pochhammer symbol and its applications to hypergeometric functions, Appl. Math. Comput., 226 (2014), 484-491. 1, 1, 1, 1

[24] R. Srivastava, N. E. Cho, Some extended Pochhammer symbols and their applications involving generalized hypergeometric polynomials, Appl. Math. Comput., 234 (2014), 277-285. 1

[25] H. M. Srivastava, J. Choi, Zeta and q-Zeta functions and associated series and integrals, Elsevier, Inc., Amsterdam, (2012). 1, 3

[26] H. M. Srivastava, P. W. Karlsson, Multiple Gaussian hypergeometric series, Ellis Horwood Series: Mathematics and its Applications, Ellis Horwood Ltd., Chichester; Halsted Press [John Wiley \& Sons, Inc.], New York, (1985). 1, 1, $1,2.1,5$

[27] H. M. Srivastava, H. L. Manocha, A treatise on generating functions, Ellis Horwood Series: Mathematics and its Applications, Ellis Horwood Ltd., Chichester; Halsted Press [John Wiley \& Sons, Inc.], New York, (1984). 1, 2.1, 3,5

[28] H. M. Srivastava, R. K. Parmar, P. Chopra, A class of extended fractional derivative operators and associated generating relations involving hypergeometric functions, Axioms, 1 (2012), 238-258. 1

[29] H. M. Srivastava, R. K. Parmar, M. M. Joshi, Extended Lauricella and Appell functions and their associated properties, Adv. Stud. Contemp. Math., 25 (2015), 151-165. 1, 2.1, 5 